\title{
Há aumento do peso corporal nos finais de semana e nas festas de final de ano? Análise em participantes de programa de exercício supervisionado
}

Paloma Gomes ${ }^{1}$ e Claudio Gil Soares de Araújo ${ }^{1,2}$

\section{RESUMO}

Fundamentação: Acredita-se que o peso corporal (PC) de adultos tende a aumentar fora dos dias úteis, seja pela maior ingestão ou pela redução de exercício. Objetivamos verificar se o PC aumenta nos finais de semana e nas festas de finais de ano. Métodos: Analisamos, retrospectivamente, os dados de 53 adultos (36 homens), com idade entre 38 e 81 anos (64 \pm 10$)$, freqüentando um programa de exercício supervisionado - três a seis sessões semanais de 60 minutos, incluindo exercícios aeróbicos, de fortalecimento muscular e de flexibilidade individualmente prescritos. Excluíram-se aqueles que faltaram mais de $25 \%$ das sessões, mais de duas semanas consecutivas ou que faziam uso de diuréticos ou outras medicações capazes de afetar o PC. O PC foi registrado antes de cada sessão. Foram analisados os dados registrados em duas semanas do mês de novembro e nas duas sessões pré e pós-festas de final de ano. Resultados: Houve estabilidade no PC - (média \pm erro padrão) 74,6 \pm 1,7 vs. 74,6 \pm 1,7kg -, respectivamente, antes e após o final de semana $(p=0,382)$, enquanto houve um aumento pequeno, porém significativo, com as festas de final de ano - 74,3 $\pm 1,7$ vs. 74,9 $\pm 1,7 \mathrm{~kg}-(p<0,001)$. Ganhos superiores a 1 e $2 \%$ do PC (máximo individual de 3,8\%) com as festas de final de ano foram observados, respectivamente, em apenas nove $(16,9 \%)$ e seis $(11,3 \%)$ dos indivíduos, com maiores variações absolutas nos indivíduos mais pesados. Discussão: Em adultos participantes de um programa de exercício supervisionado, o impacto do final de semana e das festas de final de ano sobre o PC é nenhum ou pequeno e provavelmente restrito a apenas uma parcela pequena dos homens mais pesados. Provavelmente o estilo de vida mais saudável permite com que não haja um desequilíbrio importante entre a ingestão de calorias e o dispêndio de energia pelo exercício físico nas circunstâncias estudadas.

\section{RESUMEN}

\section{¿Hay aumento del peso comporal en las fiestas de final de año? Análisis en participantes de un programa de ejercicio supervi- sado}

Fundamentación: Se dice que el peso corporal de los adultos tiende a aumentar fuera de los días útiles, ya sea por la mayor ingestión, o por la reducción del ejercicio. El objetivo es fundamentar si el peso corporal aumenta en los fines de semana y en las fiestas de fin de año. Métodos: Analizamos restrospectivamente los datos de 23 adultos (36 hombres) con edades entre 38 y 81

1. Clinimex - Clínica de Medicina do Exercício.

2. Programa de Pós-Graduação em Educação Física da Universidade Gama Filho, Rio de J aneiro, RJ .

Recebido em 17/12/03. 2a versão recebida em 26/1/04. Aceito em 1/4/04.

Endereço para correspondência: Dr. Claudio Gil Soares de Araújo, Clinimex (www. clinimex.com.br), Rua Siqueira Campos, 93/101 - 22031-070 Rio de J aneiro, RJ - Brasil. E-mail: cgaraujo@iis.com.br

\begin{abstract}
Palavras-chave: Peso corporal. Exercício supervisionado. Sarcopenia. Exercício físico. Reabilitação cardíaca.

Palabras-clave: Peso corporal. Ejercicio supervisado. Sarcopenia. Ejercício físico. Rehabilitación cardíaca.
\end{abstract}

años (64 \pm 10 ), frecuentando un programa de ejercicio de tres a seis sesiones semanales de 60 minutos con ejercicios aeróbicos, de fortalecimiento musculary de flexibilidad individualmente prescriptos. Se excluyeron aquellos que faltaron a mas del $25 \%$ de las sesiones, mas de dos semanas consecutivas o que usaran diuréticos $u$ otras medicaciones capaces de afectar el peso corporal. El peso corporal fue registrado antes de cada sesión, siendo registrado en dos semanas del mes de noviembre y en dos sesiones pre y post dias de las fiestas de fin de año. Resultados: Hubo estabilidad en el peso corporal (media \pm error padrón) 74,6 6 1,7 vs. 74,6 $\pm 1,7 \mathrm{~kg}-$, respectivamente, antes y después del final de semana ( $p=0,382)$, en cuanto hubo un aumento pequeño, por significativo, con las fiestas de fin de año - 74,3 $\pm 1,7 \mathrm{vs}$. 74,9 $\pm 1,7 \mathrm{~kg}-(p$ $<0,001)$. Los aumentos superiores a 1 e $2 \%$ del peso corporal (máximo individual de 3,8\%) con las fiestas de fin de año fueron observados, respectivamente, en apenas 9 (16,9\%) y 6 (11,3\%) dos individuos, com mayores variaciones absolutas los individuos mas pesados. Discusión: En adultos participantes de un programa de ejercicio supervisado, el impacto final del fin de semana o de las fiestas de fin de año sobre el peso corporal es ninguno o pequeño y probablemente restringido a una porción muy pequeña de dos hombres muy pesados. Probablemente el estilo de vida saludable permite que no halla un desequilibrio importante entre la ingestión de calorías y la utilización de energía por el ejercicio físico en las circunstancias estudiadas.

\section{INTRODUÇÃO}

Dentre as variáveis biológicas, o peso corporal talvez seja a mais comumente medida. Normalmente, acredita-se que a manutenção do peso corporal no adulto dentro de limites estreitos represente um sinal de boa saúde. Enquanto no início da vida adulta parece haver um equilíbrio entre a ingestão de calorias e o gasto energético, com o passar dos anos, primariamente em função da perda da massa muscular e da força (quadro clínico denominado de sarcopenia) e conseqüentemente redução do metabolismo basal, esse equilíbrio tende a desaparecer e surge um predomínio relativo do componente de consumo calórico, levando a um incremento lento e progressivo do peso corporal. Este aumento é proporcionalmente maior de gordura corporal, que tende a se acumular na região central do corpo. Dados recentes sugerem que há mais de um bilhão de indivíduos com excesso de peso ou obesidade, alcançando níveis majoritários em algumas populações, como a norte-americana ${ }^{(1,2)}$.

Exercício físico regular e dieta hipocalórica têm sido utilizados com êxito para a redução dos níveis de obesidade e de algumas de suas co-morbidades associadas, como a dislipidemia e a síndrome metabólica ${ }^{(2,3)}$. Particularmente, causam preocupação adicional a 
irregularidade e a compulsão alimentar que levam a grandes variações de peso corporal em curto período de tempo, situações essas nas quais o exercício físico regular também parece oferecer benefício, especialmente se associado à terapia cognitiva comportamental $(4,5)$.

Muitos dos praticantes ou candidatos à prática de programas regulares de exercício físico almejam uma redução do peso corporal. Em geral, participantes deste tipo de programas, com ou sem orientação nutricional especializada, tendem a auferir benefícios de saúde, incluindo a possibilidade de redução de peso e de gordura corporal(6). Contudo, o acompanhamento do peso corporal pode se tornar difícil em função de sua variabilidade no cotidiano, decorrente de mudanças no estado de hidratação e de alimentação do indivíduo. Em adendo, a medida do peso corporal pode ser avaliada utilizando diferentes e variados protocolos.

O nosso padrão alimentar tende a variar especialmente entre finais de semana e dias úteis ${ }^{(7,8)}$. Naqueles que freqüentam habitualmente um programa de exercício durante a semana, há um receio de que nos finais de semana, especialmente naqueles que não conseguem ou não podem se exercitar nesses dias possa ocorrer um aumento do peso corporal, com uma tendência inversa no decorrer da semana, dias em que eles seriam fisicamente mais ativos e com menor tendência a exageros alimentares. Essas eventuais mudanças desfavoráveis de peso corporal poderiam ser ainda maiores durante períodos de festividades, especialmente nas festas de final de ano.

Considerando a relevância prática dessas questões, objetivamos estudar se de fato há aumento no peso corporal durante os finais de semana e com as festas de finais de ano em indivíduos adultos participantes de um programa de exercício supervisionado.

\section{MATERIAIS E MÉTODOS}

O presente trabalho caracterizou-se por um estudo de seguimento, em que foram analisados retrospectivamente indivíduos que freqüentaram regularmente um programa de exercício supervisionado - três a seis sessões semanais de aproximadamente uma hora, incluindo 30 a 40 minutos de exercícios aeróbicos, duas séries de seis a oito repetições de oito a doze exercícios de fortalecimento muscular e de uma série de exercícios de flexibilidade predominantemente estáticos, sempre individualmente prescritos. A prescrição era inicialmente baseada na condição clínica do indivíduo determinada a partir de uma avaliação médica inicial específica para o programa de exercício supervisionado, que incluía um teste cardiopulmonar de exercício com determinação do limiar anaeróbico. Posteriormente, essa prescrição passava a ser reavaliada com base nos resultados das sessões anteriores e na condição clínica do indivíduo que antecedia a realização da sessão de exercício.

Somente foram incluídos os indivíduos que participaram de pelo menos oito sessões mensais de exercício nos meses de novembro e dezembro (antes das festas) de 2002, as duas primeiras sessões de janeiro de 2003 e que não se ausentaram por mais de 15 dias do programa durante o período do estudo. Foram ainda excluídos aqueles que estavam em uso de diuréticos ou outras medicações destinadas especificamente a reduzir o peso corporal e ainda aqueles que pesassem mais do que $150 \mathrm{~kg}$, fora da faixa de medida da nossa balança. Foram assim incluídos dados de 53 indivíduos (36 homens e 17 mulheres), com idade entre 38 e 81 anos $(64 \pm 10)$.

O peso corporal foi registrado no início de cada sessão, com resolução de $0,1 \mathrm{~kg}$, usando sempre a mesma balança Personal Line (Filizola, Brasil) e nas mesmas condições de vestimenta. Para efeito de análise estatística foram objetivamente identificados: a) para comparação dos pesos corporais pré e pós-finais de semana, os dados da última e da primeira sessão de exercício em duas semanas consecutivas e calculada, respectivamente, a média dos mesmos e b) para comparação dos pesos corporais pré e pós-festas de final de ano. Diferenças entre as médias das medidas de peso corporal foram testadas pelo teste-t emparelhado e coeficientes de correlação momento-produto de Pearson foram calculados quando apropriado. Para todos os procedimentos estatísticos foi aceito 5\% como nível de significância.

\section{RESULTADOS}

Houve estabilidade no peso corporal - (média \pm erro padrão) 74,6 0 1,7 vs. 74,6 \pm 1,7kg -, respectivamente, antes e após o final de semana ( $p=0,382$ ) (figura 1 ), enquanto houve um aumento pequeno, porém significativo, do peso corporal após as festas de final de ano - 74,3 $\pm 1,7 \mathrm{vs} .74,9 \pm 1,7 \mathrm{~kg}-(\mathrm{p}<0,001)$ (figura 2). Do total de indivíduos estudados, cerca de $70 \%(n=37)$ ganharam peso com as festas de final de ano.

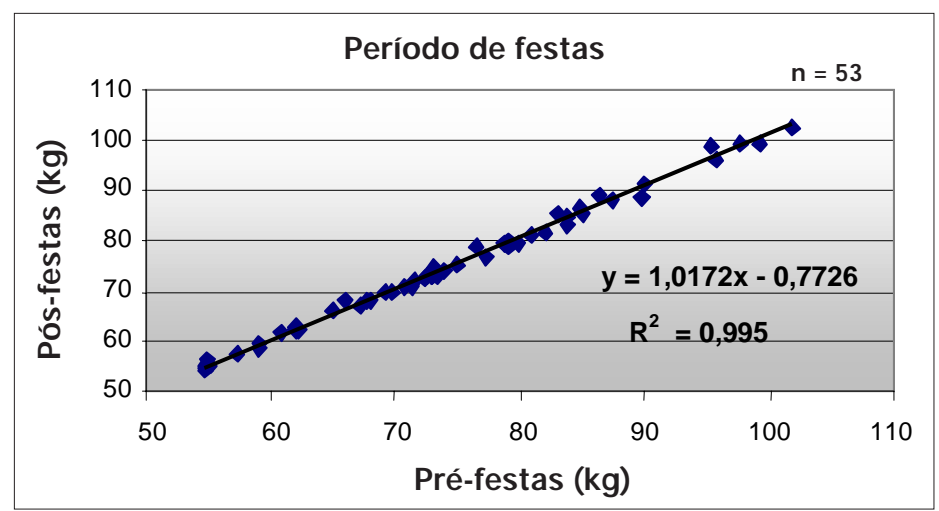

Fig. 1

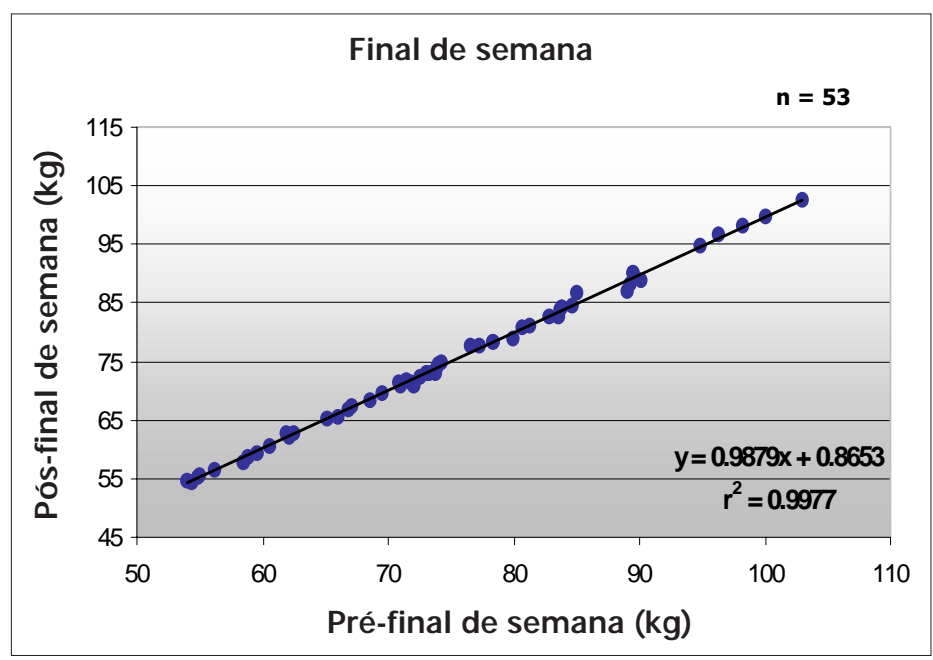

Fig. 2

Ganhos superiores a 1 e $2 \%$ do peso corporal (máximo individual de $3,8 \%$ ) com as festas de final de ano foram observados, respectivamente, em nove $(16,9 \%$ ) e seis $(11,3 \%)$ indivíduos, ocorrendo maiores variações absolutas nos indivíduos mais pesados. Os ganhos superiores a $1 \%$ foram muito mais comuns nos homens do que nas mulheres - 32\% $(n=12)$ vs. $8 \%(n=3)$, enquanto não se caracterizou uma relação com a idade.

\section{DISCUSSÃO}

O delineamento experimental mais valorizado cientificamente é o que contempla randomização dos indivíduos, existência de um grupo controle adequado e a apresentação duplo-cego. Contudo, 
muitas vezes, esse delineamento não é viável ou pode até mesmo ser prejudicial à realização e interpretação do estudo. Variáveis relacionadas ao estilo de vida, tais como, hábitos e atitudes alimentares e de exercício, são moduladas voluntariamente pelos indivíduos, podendo ser fortemente influenciadas por ações externas. No nosso caso específico, uma abordagem prospectiva em indivíduos participantes e não-participantes de um programa de exercício supervisionado sobre medidas do peso corporal introduziria uma variável relevante e de controle individualmente não-quantificável. Dessa forma, optamos por um delineamento retrospectivo, no qual pudéssemos selecionar cuidadosamente os indivíduos que atendiam determinados critérios e assim eliminando o provável efeito do conhecimento prévio de que medidas repetidas seriam realizadas, sobre o peso corporal antes e após finais de semana ou festas de fim de ano. Com essa estratégia, perdemos a possibilidade de ter um grupo controle e assim comparar o impacto da participação em um programa de exercício medicamente supervisionado com a ausência de participação. Considerando essa limitação, os nossos resultados devem ser analisados como algo que pôde ser observado em indivíduos participantes de um programa, sem qualquer ilação sobre o que poderia ter acontecido com indivíduos que não participam de um programa dessa natureza.

Contrariando a expectativa inicial, os nossos resultados indicaram uma manutenção do peso corporal dos indivíduos após os finais de semana, o que provavelmente pode ser explicado pela prática regular de exercício físico e maior atenção e cuidado com a dieta, e conseqüentemente hábitos de vida mais saudáveis mesmo nos dias em que eles não participavam do programa de exercício supervisionado. Em uma abordagem um pouco distinta, Schairer et al. ${ }^{(9)}$ também observaram que participantes de programas de exercício tendem a manter-se fisicamente mais ativos durante as horas de lazer. Em adendo, pode-se esperar que as habituais variações alimentares típicas de dias úteis e finais de semana ${ }^{(7,10)}$ não tendam a ocorrer de modo apreciável em nossos indivíduos que participam de um programa de exercício supervisionado.

Por outro lado, o aumento pequeno, porém significativo, do peso corporal com as festas de final de ano pode ter ocorrido devido a uma redução na prática de exercício físico e de um "tradicional" aumento na ingestão calórica associada à esses eventos festivos. É interessante observar que nesse contexto, os homens tenderam a apresentar um maior aumento do peso corporal quando comparados às mulheres, o que talvez possa ser devido a uma menor ingestão calórica por parte das mulheres, função de uma maior preocupação das mulheres com a manutenção do peso corporal e de sua estética(11). Já os indivíduos mais pesados apresentaram uma maior variação do peso corporal, provavelmente por terem uma maior dificuldade em manter a ingestão calórica no período das festas, quando comparado aos dias em que freqüentam o programa de exercício supervisionado.

\section{REFERÊNCIAS}

1. Prevalence of physical activity, including lifestyle activities among adults - United States, 2000-2001. MMWR Morb Mortal Wkly Rep 2003;52:764-9.

2. Keller KB, Lemberg L. Obesity and the metabolic syndrome. Am J Crit Care 2003;12:167-70.

3. Lalonde L, Gray-Donald K, Lowensteyn I, Marchand S, Dorais M, Michaels G, et al. Comparing the benefits of diet and exercise in the treatment of dyslipidemia. Prev Med 2002;35:16-24.

4. Levine MD, Marcus MD, Moulton P. Exercise in the treatment of binge eating disorder. Int J Eat Disord 1996;19:171-7.

5. Pendleton VR, Goodrick GK, Poston WS, Reeves RS, Foreyt J P. Exercise augments the effects of cognitive-behavioral therapy in the treatment of binge eating. Int J Eat Disord 2002;31:172-84.

6. Friedman DB, Williams AN, Levine BD. Compliance and efficacy of cardiac rehabilitation and risk factor modification in the medically indigent. Am J Cardiol 1997;79:281-5.
Ainda que a medida do peso corporal e a participação no programa de exercício tenham sido bem controladas, esse estudo apresenta uma série de limitações metodológicas. Inicialmente, não foi controlada ou argüida a dieta ou a prática de exercício físico e atividade física fora do programa. Também não foi controlado o tempo de participação anterior no programa de exercício, já que a amostra estudada incluía indivíduos com tempos de participação entre vários anos e algumas poucas semanas. Em adendo, não foram obtidas medidas mais precisas de composição corporal ou de perfil laboratorial que pudessem ampliar as implicações ou interpretações dos resultados observados. Particularmente, teria sido de interesse observar se os nossos indivíduos, aparentemente com atitudes adequadas quanto a exercício e dieta nos dias em que não freqüentam o programa de exercício supervisionado, apresentavam as mesmas pioras encontradas em valores de laboratório para medidas colhidas nas segundas-feiras quando comparadas aos outros dias da semana nos pacientes estudados por Urdal et al.(8).

\section{CONCLUSÃO}

Apesar das limitações elencadas acima, conclui-se que em adultos participantes de um programa de exercício supervisionado o impacto do final de semana e das festas de final de ano sobre o peso corporal é nenhum ou pequeno e provavelmente restrito a apenas uma parcela pequena dos homens mais pesados. Acredita-se que o estímulo para a adoção de um estilo de vida saudável atual nesses indivíduos permite com que não haja um desequilíbrio importante entre a ingestão de calorias e o dispêndio de energia pelo exercício físico nas circunstâncias estudadas.

Outra possibilidade teórica para os nossos resultados é que a prática regular de exercícios aeróbicos e de fortalecimento muscular, minimize a sarcopenia normalmente observada nos indivíduos dessa faixa etária(12), o que, em tese, poderia facilitar a manutenção de um metabolismo mais alto mesmo nos dias em que não é feito exercício físico, reduzindo a possibilidade de ganho apreciável de peso corporal.

Finalmente, deve-se enfatizar que esses resultados foram obtidos em indivíduos que praticam regularmente exercício físico. A generalização destes dados para a sociedade não é possível, uma vez que não estudamos um grupo controle formado por indivíduos sedentários. Acredita-se que, com o aumento dos níveis de prevalência de sedentarismo e de obesidade, devam ser esperadas maiores variações no peso corporal. Uma sugestão de estudo seria comparar o comportamento do peso corporal nessas situações entre indivíduos fisicamente ativos e sedentários.

Todos os autores declararam não haver qualquer potencial conflito de interesses referente a este artigo.
7. Tarasuk V, Beaton GH. Statistical estimation of dietary parameters: implications of patterns in within-subject variation - a case study of sampling strategies. Am J Clin Nutr 1992;55:22-7.

8. Urdal P, Anderssen SA, Holme I, Hjermann I, Mundal HH, Haaland A, et al. Monday and non-monday concentrations of lifestyle-related blood components in the Oslo Diet and Exercise Study. J Intern Med 1998;244:507-13.

9. Schairer J R, Keteyian SJ , Ehrman J K, Brawner CA, Berkebile ND. Leisure time physical activity of patients in maintenance cardiac rehabilitation. J Cardiopulm Rehabil 2003;23:260-5.

10. Post B, Kemper HC, Storm-Van Essen L. Longitudinal changes in nutritional habits of teenagers: differences in intake between schooldays and weekend days. BrJ Nutr 1987;57:161-76.

11. Araújo DSMS, Araújo CGS. Autopercepção e insatisfação com o peso corporal independem da freqüência de atividade física semanal. Arq Bras Cardiol 2003;80:235-49.

12. Marcell TJ . Sarcopenia: causes, consequences, and preventions. J Gerontol A Biol Sci Med Sci 2003;58:M911-6. 